\title{
A view of three different lipid monolayers by atomic force microscopy
}

\author{
A. Ortiz, M.A. Alsina and Y. Cajal \\ Department of Physical Chemistry, Faculty of Pharmacy, University of Barcelona, \\ Avn. Joan XXIII, s/n, 08028 Barcelona, Spain
}

\begin{abstract}
Lipid monolayers prepared by the Langmuir technique are commonly used as model biomembranes. They have been extensively studied for years with a wide range of techniques, and during the last decade atomic force microscopy (AFM) [1] has emerged as a powerful tool for imaging the structure of the supported lipid films. AFM offers the view at nanometric scale of the transferred monolayers which provides information on the topographical organization of the films [2-4]. In the present work we have studied the morphology of three lipid monolayers: zwitterionic DPPC, anionic DPPC:PI (9:1, mol:mol) and cationic DPPC:SA. (9:1, mol:mol).The structure of these three mixed monolayers transferred on mica is determined by AFM, and compared with the phase properties of the films at the air-water interface.
\end{abstract}

\section{EXPERIMENTAL SECTION}

\subsection{Chemicals}

The phospholipids used in the study were 1,2-dipalmitoylphosphatidylcholine (DPPC), phosphatidylinositol from soy bean (PI), and the fatty acid stearylamine (SA) from Sigma Chemical Co. The purity was checked by thin-layer chromatography. Chloroform (HPLC grade from Fisher Scientific Co.) was used as the spreading solvent for all lipids. Water was double destilled and deionized (Mili-Q system, Millipore Corp.). Lipids were spread on water subphase at $25^{\circ} \mathrm{C}$.

\subsection{Methods}

\subsubsection{Compression Isotherms}

The compression isotherms were performed on a Langmuir film balance KSV5000 equipped with a Wilhelmy platinum plate. The PTFE (surface area $17000 \mathrm{~mm}^{2}$, volume $1000 \mathrm{~cm}^{3}$ ) and the plate were thoroughly cleaned before each run with hot water and $\mathrm{Cl}_{3} \mathrm{CH}: \mathrm{CH}_{3} \mathrm{OH}(2: 1 \mathrm{v} / \mathrm{v})$, to avoid carry over of lipid. Lipid mixtures for spreading were prepared by premixing appropriate amounts of the components from stock solutions, so that the final concentration was $1.36 \mathrm{mM}$. Monolayers were formed by adding small drops of the solutions on the water subphase, with a microsyringe ( Hamilton Co., Reno, NV). After 10 minutes, the monolayers were compressed (symmetrical compression) with an area reduction rate of $60 \mathrm{~mm}^{2} / \mathrm{min}$, up to their collapse pressure. The temperature of the subphase was $25 \pm 1^{\circ} \mathrm{C}$. Each run was repeated three times, and reproducibility was $\pm 0.05 \mathrm{~nm}^{2} /$ molecule.

\subsubsection{Langmuir - Blodgett Film. Atomic Force Microscopy}

In order to investigate the morphology of the LB films with different composition, films at $15 \mathrm{mN} / \mathrm{m}$ surface pressure were transferred onto mica (Hydrophilic substrate). The preparation of the Langmuir Blodgett film was performed using a KSV5000 balance. The subphase was high purity water (MiliQ, 
electrical resistivity> $18 \mathrm{M} \Omega \mathrm{cm}$, at $\mathrm{pH} 6.8$ ) with a temperature $25 \pm 1^{\circ} \mathrm{C}$. Phospholipid solution was spread, drop by drop. The compression barrier speed was $5 \mathrm{mN} \cdot \mathrm{m}^{-1} \cdot \mathrm{min}^{-1}$ and dipping speed was $0.1 \mathrm{~mm} \cdot \mathrm{min}^{-1}$ for all experiments. The transfer ratios were T.R. $\cong 1$ for all experiments.

The technique consists on scanning the sample surface with a sharp probing tip, fixed at the end of a cantilever, and, using an optical method, to measure the deflection of the cantilever due to the forces between it and the sample surface during the scan. The displacement in three dimensions is provided by a high sensitive piezoelectric scanner. We used the tapping mode (TM-AFM), where the tip is forced to oscillate in the $\mathrm{z}$ direction above the sample and touches its surface periodically. The Atomic Force Microscope (AFM) used was a Nanoscope III Extended Multimode from Digital Instruments and the tips (Point Probes, Nanosensors, Wetzlar-Blankenfeld, Germany) were in silicon monocristaline with a spring constant of 38-74 N/m and a resonance frequency between $270-377 \mathrm{kHz}$. Mica substrate used was ASTM-V2 Green mica (Asheville-Schoonmaker Mica Co, Virginia, USA). Images at high resolution were taken on the low edge of the sample.

\section{RESULTS}

\subsection{Compressibility studies}

The comprenssion isotherms of different lipid mixtures of two components selected from a previous experiments, DPPC, DPPC-PI (9:1), DPPC-SA (9:1), were obtained, and the plots of surface pressure $(\pi)$ vs mean molecular area at $25^{\circ} \mathrm{C}$ are shown in figure $1 \mathrm{~A}$. The three monolayers have a different behaviour, but they show the presence of liquid expanded and liquid condensed phases as the film is compressed except for the anionic monolayer. This one is in a more expanded state. The cationic monolayer presents the lowest area per molecule trough most of the surface pressure range. The compressibility modulus as a function of the molecular area of the mixed films are represented in the figure 1B. The compressibility modulus is defined as

$$
C_{S}^{-1}=-A\left(\frac{\partial \pi}{\delta A}\right)_{T}
$$

Its value can be used to characterise the phase behaviour of a monolayer, according to Davies and Rideal [5], where for liquid expanded state (LE) values range between 12.5 and $50 \mathrm{mN} / \mathrm{m}$, and for liquid condensed (LC) phase compressibility modulus is $100<\mathrm{C}_{\mathrm{s}}^{-1}>250$.
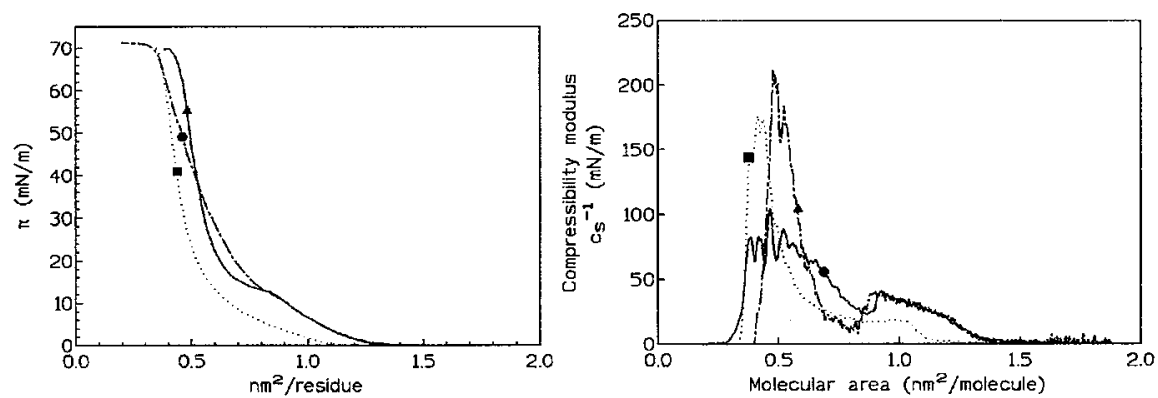

Figure 1. Isotherms of surface pressure versus mean molecular area for monolayers of DPPC (A) DPPC/PI (•) and DPPC/SA (E) on Subphase of water at $25^{\mathrm{a}} \mathrm{C}$. Compressibility modulus versus mean molecular area for the same monolayers.

The cationic monolayer is in a liquid-condensed phase at molecular areas below $0.49 \mathrm{~nm} / \mathrm{molecule}$, above this value and up to $0.55 \mathrm{~nm}^{2} /$ molecule is in a liquid intermediate phase, and for bigger areas the 
monolayer adopts liquid-expanded state. In the case of the DPPC monolayer, $\mathrm{C}_{\mathrm{s}}^{-1}$, indicates the presence of a liquid-condensed phase at areas below $0.58 \mathrm{~nm}^{2} /$ molecule, a liquid-intermediate phase between 0.58 and $0.63 \mathrm{~nm}^{2} /$ molecule, and liquid-expanded phase at molecular areas above $0.63 \mathrm{~nm}^{2} / \mathrm{molecule}$. Finally, the anionic monolayer, $\mathrm{C}_{\mathrm{s}}^{-1}$ values show a liquid-expanded phase above $0.7 \mathrm{~nm}^{2} /$ molecule and a liquidintermediate state at smaller areas, without passing through a liquid-condensed phase before the collapse. These values are interesting for description of the monolayer behaviour, to check the miscibility of the components in the monolayer at air-water interface and to help on the comprehension of the topographic images taken by AFM tapping mode.

\subsection{AFM results}

The three monolayers, DPPC-PI (9:1), DPPC, DPPC-SA (9:1), at deposition pressure of $15 \mathrm{mN} / \mathrm{m}$ and $25^{\circ} \mathrm{C}$, occupy $0.795,0.664$ and $0.554 \mathrm{~nm}^{2} /$ molecule respectively. Their $\mathrm{Cs}^{-1}$ are between $50 \mathrm{mN} / \mathrm{m}$ and $100 \mathrm{mN} / \mathrm{m}$, indicating that the three monolayers are in a liquid intermediate (LI) state and the molecules are not in a fully extended length.

Figure $2 \mathrm{~A}$ is a zoom area of a typical AFM image of a fresh DPPC film (measured within $2 \mathrm{~h}$. after deposition) transferred in the beginning of LC phase. Three features are seen here:1-The LC domains, namely macrodomains; there are three typical shapes globular, lobular and ovoid for the big, intermediate and the small LC domain respectively. Outside LC domains, numerous microdomains like grains and clusters of grains are observed.2- the macrodomain boundaries are not smooth but exhibit irregular structures.3- Holes like defects are observed within the macrodomains, particularly for the globular at the edge region. The height of the macrodomain is $0.984 \pm 0.05 \mathrm{~nm}$, the width of the intermediate and small macrodomains are $314.4 \pm 27.3 \mathrm{~nm}$ and $168.7 \pm 59.3 \mathrm{~nm}$, respectively, while the diameter for holes is $48.83 \pm 5.86 \mathrm{~nm}$. In the DPPC AFM image, LE and LC phase coexist. This observation is in agreement with other reported previously, where LE and LC phase were observed at least to the onset of collapse [6]. The LC domains are not present at air/water interface, but they are probably induced in the transferred film by the thinning of the water layer between the lipid and the mica surface [7-8].

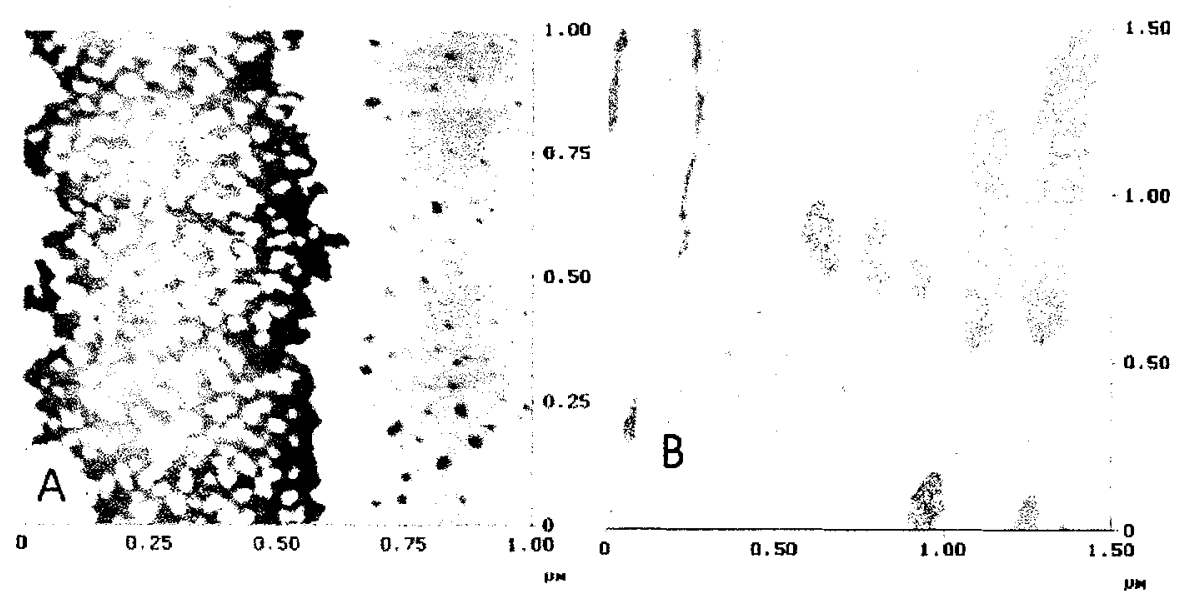

Figure 2. AFM tapping mode images of (A) DPPC and (B) DPPC/SA (9:1) monolayers deposited at $15 \mathrm{mN} / \mathrm{m}$ on mica by Langmuir-Blodgett deposition. Length scales for DPPC and DPPC/SA (9:1) are as follows: (A) $1 \mu \mathrm{m} \times 1 \mu \mathrm{m}$; (B) 1.5 $\mu \mathrm{m} \times 1.5 \mu \mathrm{m}$.

DPPC-SA (9:1) monolayer observed by AFM (figure 2B) shows a homogenous and practicaly complete matrix with some irregular discontinuites and different circular holes within the film. The height of this 
matrix is $0.671 \pm 0.03 \mathrm{~nm}$ and the diameter of the big holes is $156.2 \pm 37.1 \mathrm{~nm}$, and for small ones is $75.8 \pm 15.2 \mathrm{~nm}$. Almost all lipids are presented in a continuos LC domain, as stearylamine is a saturated large fatty acid that at the $\mathrm{pH}$ of the experiments is positively charged, probably is more attached to the mica surface and better compacted with the DPPC molecules due to the cohesive Van der Waals forces that hink the chains up.

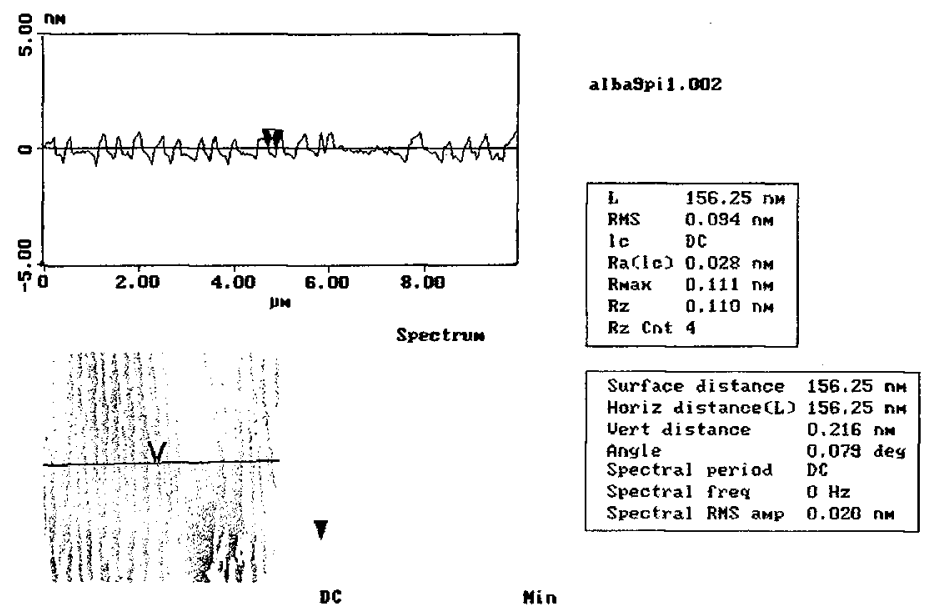

Figure 3. AFM tapping mode images of DPPC/PI (9:1) monolayers deposited at $15 \mathrm{mN} / \mathrm{m}$ on mica by Langmuir-Blodgett deposition. Length scales DPPC/PI $(9: 1)$ is $10 \mu \mathrm{m} \times 10 \mu \mathrm{m}$.

DPPC-PI monomolecular film seemed soft under AFM tip and it is always difficult to get a stable image [9], due to its negative charge. That is the reason we take images of this monolayer 100 times diluted. An inhomogeneous monolayer is observed with long longitudinal islands where the amplitude is $286 \pm 45.6$ $\mathrm{nm}$ and the periodicity is $116.02 \pm 9.76 \mathrm{~nm}$. The height of these long macrodomains is $1.093 \pm 0.11 \mathrm{~nm}$ and inside of them there are a few elongated holes. In the anionic monolayer, the ripples or elongated macrodomains present on the mica substrate are formed by phosphatydilinositol, and are due in part to the hydrogen bonds between the polar head and the water. In addition, lower cohesion stability is expected in mixed DPPC-PI monolayers, due to reduced Van der Waals interaction between the saturated (DPPC) and unsaturated (PI) acyl chains[10]. These unsaturations present in the lipid matrix give an expansion of the LC macrodomain.

\section{References}

[1] Binning, G.; Quate, C.F.; Gerber, Ch. Phys. Rev. Lett. 56, (1986) 930 - 933

[2] Schwartz, D.K.; Garnaes, J.; Viswanathan, R.; Zasadzinski, J.A.N. Science 257, (1992) 508-511

[3] Chi, L.F.; Fuch, H.; Jonhston, R.R.; Ringsdorf, H. Thin solid film 242, (1994) 151

[4] Sikes, D.H.; Woodward, J.T.; Schwartz, D.K. J. Phys. Chem. 100, (1996) 9093-9097

[5] Davies, J.T.; Rideal, E. K.; Interfacial Phenomena, 2nd ed.; Academic Press: New York and London $1963 ;$ pp. 265.

[6] Crane,J.; Putz, G.; Stephane, B.H. J. Biophys. 77, (1999) 3134-3143

[7] Hollars, C.W.; Dunn, R.C.; J. Biophys. 75(1), (1998) 342

[8] Fang, J.; Knobler, C.M.; J.Phys.Chem. 99, (1995)10425

[9] Hilde, A.R.; Demel, A.R; Van der Eerden, J.P.J.M.; de Kruijff, Ben.J. Biophys.77(3), (1999) 16381693

[10] DeWolf, C.; Leporatti, S.; Kirsch, C.; Klinger, R.; Brezesinski, G. Chem. and Phys. of Lipids 97, (1999) 129-138 